\title{
Quantum Hall Effect In Bilayer Systems And The Noncommutative Plane: A Toy Model Approach
}

\author{
B. Bast田 and Subir Ghosh \\ Physics and Applied Mathematics Unit \\ Indian Statistical Institute \\ Kolkata-700108
}

\begin{abstract}
We have presented a quantum mechanical toy model for the study of Coulomb interactions in Quantum Hall $(\mathrm{QH})$ system. Inclusion of Coulomb interaction is essential for the study of bilayer QH system and our model can simulate it, in the compound state, in a perturbative framework. We also show that in the noncommutative plane, the Coulomb interaction is modified at a higher order in the noncommutativity parameter $\theta$, and only if $\theta$ varies from layer to layer in the QH system.

PACS numbers: 73.43.-f, 03.65.-w
\end{abstract}

Physical conditions for the occurrence (as well as experimental observation) of Integer Quantum Hall Effect (IQHE) 1] allows us to reduce the many electron system to an effective single electron one. This miraculous simplification is possible due to the incompressibility of the planar electron gas in the presence of a perpendicular high magnetic field at very low temperature and without disorder [1]. The incompressibility of the ground state forbids the creation of separated particle-hole pairs, (capable of carrying current), because their creation requires energy. This is the key point of the quantum Hall system which at the same time suggests the usage of single particle picture. It can be explicitly shown that quantum mechanics of a single electron in a magnetic field is able to capture the discrete nature of Hall conductivity. Indeed, this simple model can not address most of the details of $\mathrm{QH}$ physics, notably among them formation of the Hall plateauxs, the (passive) role of disorder and impurities etc. (see for example Stone in $[1]$ ).

Compared to IQHE, which is essentially a one body effect, explanation of Fractional Quantum Hall Effect (FQHE) 1] requires the introduction of many body physics since the latter is induced by interparticle forces, Coulomb interactions (between electrons confined to the two-dimensional plane) being the dominant one. The magnetic field can be large enough to effectively freeze out both the kinetic and spin Zeeman energies. In [2] an explanation of the $\nu=\frac{1}{2 n+1} \mathrm{FQH}$ states was given. In an attractive alternative approach, quantum Hall effect was also studied in spherical geometry [3] which was pursued [4] in the Berry phase framework. This formalism can nicely [5] accommodate the newly observed [ 6$]$ FQH states within the primary sequence. The concept of composite particles play an important role in understanding the mechanism of incompressibility in FQHE. This last fact motivates us to question how far one can pursue the study of a planar many body interacting system in the $\mathrm{QH}$ regime, in terms of single particle states the describe IQHE. An interesting idea, mooted in [9], is that FQHE might be interpreted as IQHE in the Non-Commutative (NC) plane, where the $\mathrm{NC}$ parameter $\theta$ generates the fractional QH states. In the present Letter, we first elaborate our proposed toy model for a bilayer QH system and subsequently consider the effects of the NC coordinates onit. Hopefully the method and results presented here can be generalized to study a restricted class of systems exhibiting FQHE.

Coulomb interactions are essential in the study of bilayer QH systems: a new variety of QH states. [7] A bilayer system is made by trapping electrons in two thin layers at the interface of semiconductors. There exists a variety of $\mathrm{QH}$ states depending on the relative strength of various interactions. The key interactions are the inter-layer Coulomb interaction $\left(e^{2} / 4 \pi \epsilon \sqrt{r^{2}+d^{2}}\right)$, the intra-layer Coulomb interaction $\left(e^{2} / 4 \pi \epsilon r\right)$, where $r$ is the planar inter-particle distance and $d$ is the inter-layer distance. Typical Coulomb energy scales are $e^{2} / 4 \pi \epsilon d$ and $e^{2} / 4 \pi \epsilon l$ where $l=\frac{1}{\sqrt{e B}}$ denotes the magnetic length (in natural units $c=\hbar=1$ ). We are neglecting the Zeeman interaction and the tunnelling interaction. Our analysis is relevant in the context of the simplest of bilayer states, the compound state where charges are not transferable between the two layers. Addition of the monolayer Hall conductivities yields the bilayer Hall conductivity. For this reason, we consider the states of bilayer system as product states of

*Electronic address: banasri@isical.ac.in

${ }^{\dagger}$ Electronic address: sghosh@isical.ac.in 
two monolayer single particle states. In this setup we will compute the Coulomb energy in the lowest order of perturbation theory. Qualitative agreement between our observations and recent rigorous field theoretic results [] indicate that we are on the right track.

Recent excitement in the High Energy physics community regarding the possibility of having a NonCommutative (NC) spacetime (or space) [8] has triggered the study of effects of NC space in different systems (for a review of $\mathrm{NC}$ effects on planar theories, see [10]). In [9] it was shown that $\mathrm{NC}$ effects renormalize the magnetic field $B$ and in turn the filling factor $\nu=2 \pi \rho / \sqrt{e B}$, where $\rho$ denotes the electron density. This induces a modification in the Hall conductivity $\sigma$ which is proportional to $\nu$. We will consider these $\mathrm{NC}$ effects on our toy model of the bilayer system.

Let us start by considering a monolayer QH system, the (Lowest Landau Level) states of which are reproduced by the effective single particle states of a planar charged spinless particle in a magnetic field $B$. The Hamiltonian of the model, in a crossed background electric field $(E)$ is given by,

$$
H=\frac{1}{2 m}\left[\left(p_{x}-\frac{e B}{2 c} y\right)^{2}+\left(p_{y}-\frac{e B}{2 c} x\right)^{2}\right]+e E x,
$$

where the magnetic field is expressed in the symmetric gauge [9]

$$
\mathbf{B}=\nabla \times \mathbf{A}, \quad A_{x}=-\frac{B}{2} y, A_{y}=\frac{B}{2} x
$$

and $m$ and $e$ denote the mass and charge of the particle. (Our notations and conventions agree with [9].) In order to solve $H$, we change the variables to

$$
z=x+i y \quad, p_{z}=\frac{1}{2}\left(p_{x}-i p_{y}\right)
$$

and introduce two sets of creation and annihilation operators:

$$
\begin{gathered}
b^{\dagger}=-2 i p_{\bar{z}}+\frac{e B}{2 c} z+\lambda ; \quad b=-2 i p_{z}+\frac{e B}{2 c} \bar{z}+\lambda, \\
d^{\dagger}=-2 i p_{\bar{z}}-\frac{e B}{2 c} z ; \quad d=2 i p_{z}-\frac{e B}{2 c} \bar{z}
\end{gathered}
$$

where $\lambda=\frac{m c E}{B}$.

These two sets commute with each other and satisfy the commutation relations

$$
\left[b, b^{\dagger}\right]=2 m \omega, \quad\left[d, d^{\dagger}\right]=-2 m \omega
$$

where $\omega=\frac{e B}{m c}$ is the cyclotron frequency. The Hamiltonian can be rewritten as

$$
H=\frac{1}{4 m}\left(b^{\dagger} b+b b^{\dagger}\right)-\frac{\lambda}{2 m}\left(d^{\dagger}+d\right)-\frac{\lambda^{2}}{2 m}
$$

As expected, the electric field lifts the degeneracy. The eigenfunctions and the energy spectrum of the Hamiltonian $H$ are

$$
\begin{gathered}
\psi_{(n, \alpha)}=\phi_{n} \otimes \phi_{\alpha}=\mid n, \alpha>, \\
\phi_{n}=\frac{1}{\sqrt{(2 m \omega)^{n} n !}}\left(b^{\dagger}\right)^{n} \mid 0>; \phi_{\alpha}=\exp \left(i\left(\alpha y+i \frac{m \omega}{2} x y\right)\right), \\
E_{(n, \alpha)}=\frac{\omega}{2}(2 n+1)-\frac{\lambda}{m} \alpha-\frac{\lambda^{2}}{2 m}, \quad n=0,1,2 \ldots, \quad \alpha \in R .
\end{gathered}
$$


The generic form of Hall current, induced by the Lorentz force, is

$$
J_{i}=\frac{e^{2} \nu}{2 \pi} \epsilon_{i j} E_{j}
$$

where $\nu=2 \pi l^{2} \rho$ is the filling factor with $\rho$ the electron density. In the present case, the current is derived from the equation of motion:

$$
J_{i}=i e \rho\left[H, r_{i}\right]=\frac{e \rho}{m}\left(p_{i}+\frac{e}{c} A_{i}\right)
$$

The expectation value of $J_{i}$ in the eigen-states $\psi_{n, \alpha}$ in (6) is,

$$
<J_{x}>=0, \quad<J_{y}>=-\frac{e c \rho}{B} E .
$$

Hence one can read off the Hall conductivity $\sigma$ as,

$$
\sigma=2 \pi e^{2} \nu
$$

For a system with $n$ filled Landau levels, one gets

$$
\sigma=n\left(2 \pi e^{2} \nu\right)
$$

Although the correct quantization of the Hall conductance has been achieved, quite naturally this simplistic model is unable to address a host of phenomena related to QH effect, most notably among them the formation of Hall plateaux, (absence of) effects of disorders and impurities etc..

Our idea is to carry through this effective single particle picture as far as feasible in the realm of bilayer $\mathrm{QH}$ systems. We will model the bilayer $\mathrm{QH}$ system in the compound state as a weakly coupled system of two mono-layers with negligible tunnelling between them $(\sim$ large $d)$. We take the compound state Hamiltonian $\left(H_{C}\right)$ as,

$$
H_{C}=H_{1}\left(x_{1}, y_{1}\right)+H_{2}\left(x_{2}, y_{2}\right),
$$

where $H(x, y)$ is given in (11). This is just a combination of two decoupled mono-layers. Obviously the wave functions of the compound system will be of the form,

$$
\Psi_{C}=\left[\phi_{n_{1}}^{1} \otimes \phi_{a_{1}}^{1}\right] \otimes\left[\phi_{n_{2}}^{2} \otimes \phi_{a_{2}}^{2}\right] .
$$

This type of direct product states have appeared before in [12] in the context of electron-hole pair in a quantum Hall system, without Coulomb interaction. The Hall current $J_{(C) i}$ for the compound state is given by,

$$
J_{(C) i}=i e \rho\left[H_{C}, r_{(1) i}+r_{(2) i}\right] .
$$

Clearly the Hall conductivity for the Compound system is obtained as,

$$
\sigma_{C}=\left(n_{1}+n_{2}\right) \frac{e^{2}}{\nu}
$$

where $n_{1}$ and $n_{2}$ comes from the two mono-layers.

After this somewhat trivial rederivation of the bilayer Compound state QH conductivity we come to the interesting part: effect of the Coulomb interaction. We intend to confirm that the Coulomb interaction will not affect the conductivity in the present scenario. Subsequently we will compute the correction in energy at the lowest non-trivial order of perturbation. Quite surprisingly, our "back of the envelope" estimate agrees with the rigorous findings [7], at least structurally.

Conventionally, the Coulomb interaction in bilayer systems is decomposed in to two parts [1, [],

$$
H_{\text {Coulomb }}=\Delta H_{1}+\Delta H_{2} ; \Delta H_{1}=\frac{C_{1} e^{2}}{4 \pi \epsilon_{0}} \frac{1}{|\mathbf{x}|}, \Delta H_{2}=\frac{C_{2} e^{2}}{4 \pi \epsilon_{0}} \frac{1}{\sqrt{|\mathbf{x}|^{2}+d^{2}}},
$$


where $|\mathbf{x}|=\left[\left(x_{1}-x_{2}\right)^{2}+\left(y_{1}-y_{2}\right)^{2}\right]^{1 / 2}$. Clearly $\Delta H_{1}$ and $\Delta H_{2}$ represent monolayer and bilayer contributions respectively. $C_{1}$ and $C_{2}$ are two numerical parameters which can introduce screening effects since the charges are not in vacuum. However, we will not elaborate on them any further since we only wish to present the structure of the energy correction, and not absolute numerical values.

Before proceeding any further, the expression of Coulomb term in the Hamiltonian, as posited in (17), requires an explanation. We have borrowed this term from its (more rigorous) field theoretic counterpart which reads [11,

$$
H_{\text {Coulomb }}=\frac{1}{2} \sum_{\alpha, \beta} \int d^{2} x d^{2} y V_{\alpha \beta}(\mathbf{x}-\mathbf{y}) \rho^{\alpha}(\mathbf{x}) \rho^{\beta}(\mathbf{y}) .
$$

Here $\alpha$ and $\beta$ denote the top or bottom layer and $\rho^{\alpha}$ refers to the corresponding charge density. The potential is given by,

$$
V_{\alpha \beta}(\mathbf{x}-\mathbf{y})=\frac{e^{2}}{4 \pi \epsilon} \frac{1}{\sqrt{|\mathbf{x}-\mathbf{y}|^{2}+d_{\alpha \beta}^{2}}},
$$

with $d_{t t}=d_{b b}=0$ and $d_{t b}=d$, the inter-layer separation. Notice that this pseudospin framework, (where the electrons in top and bottom layer are distinguished by "up" or "down" pseudospin), admits the possibility of tunnelling of electrons between the layers, apart from their static interactions. As we mentioned in the beginning, in our toy model approach the tunnelling of electrons between layers is not considered. Since we concentrate on the static energy, the bilayer nature of the system is manifested only through $d$. This is as if the bilayer system is projected on to a single layer. The intra and inter-layer Coulomb terms differ by the appearance of $d$ which introduces a lower bound in the inter particle distance in the inter-layer Coulomb effect.

Indeed, deduction of the first result is easy. The definition of the Hall current (9) shows that $J_{C}$ will remain unaltered since the position operators will commute with the Coulomb interaction terms (17).

Next we compute the energy correction,

$$
\Delta E_{C}=<\psi_{C}\left|\Delta H_{1}\right| \psi_{C}>+<\psi_{C}\left|\Delta H_{2}\right| \psi_{C}>,
$$

in the first perturbative order only. In terms of the creation and annihilation operators we express the coordinates as,

$$
x_{\alpha}=\frac{1}{2 e B}\left(b_{\alpha}+b_{\alpha}^{\dagger}-d_{\alpha}-d_{\alpha}^{\dagger}-2 \lambda\right), \quad y_{\alpha}=-\frac{i}{2 e B}\left(b_{\alpha}^{\dagger}-b_{\alpha}-\left(d_{\alpha}^{\dagger}-d_{\alpha}\right),\right.
$$

where $\alpha=1,2$ indicate the two mono-layers. The inter-particle distance can now be expressed in terms of the ladder operators:

$$
\begin{gathered}
\mathbf{x}^{2}=\left(x_{1}-x_{2}\right)^{2}+\left(y_{1}-y_{2}\right)^{2} \\
=\frac{1}{e^{2} B^{2}}\left[b_{1}^{\dagger} b_{1}+b_{2}^{\dagger} b_{2}+\frac{1}{2}\left\{\left(d_{1}+d_{2}^{\dagger}\right)^{2}-\left(d_{1}-d_{2}^{\dagger}\right)^{2}\right\}+2 m \omega+D\right],
\end{gathered}
$$

where

$$
D=b_{1}^{\dagger} d_{2}+d_{1}^{\dagger} b_{2}+b_{1} d_{2}^{\dagger}+d_{1} b_{2}^{\dagger}-b_{1}^{\dagger} b_{2}-b_{1} b_{2}^{\dagger}-d_{1}^{\dagger} d_{2}-d_{1} d_{2}^{\dagger}-b_{1}^{\dagger} d_{1}-d_{1}^{\dagger} b_{1}-b_{2}^{\dagger} d_{2}-d_{2}^{\dagger} b_{2}
$$

A simple calculation yields

$$
<\psi_{C}\left|\Delta H_{1}\right| \psi_{C}>=\frac{C_{1} e^{2}}{4 \pi \epsilon_{0}} e B<\psi_{C}\left|\frac{1}{\left[2 m \omega(2 n+1)+2 \alpha^{2}+\left(\frac{e B}{c}\right)^{2}\left(y_{1}^{2}+y_{2}^{2}\right)+D\right]^{1 / 2}}\right| \psi_{C}>
$$

Let us first put forward our results. For large $n$ the above relation can be approximated to yield,

$$
<\psi_{C}\left|\Delta H_{1}\right| \psi_{C}>\cong \frac{C_{1} e^{2}}{4 \pi \epsilon_{0}} e B \frac{1}{\sqrt{A}}\left(1-\frac{\alpha^{2}}{2 A}-\frac{2 \pi l^{2} e^{2} B^{2}}{3 A}\right)
$$


where $A=2 m \omega(2 n+1) \cong 4 n m \omega$. Notice that in the first order of perturbation $\left\langle\psi_{C}|D| \psi_{C}\right\rangle=0$. The other point to note is that $y$-operator matrix elements are integrated to give $2 \pi l^{2}$, the effective area of confinement of the electrons. The above result is simplified to give,

$$
<\psi_{C}\left|\Delta H_{1}\right| \psi_{C}>\cong \frac{C_{1} e^{2}}{4 \pi \epsilon_{0}}\left[\frac{1}{2 \sqrt{n}} \frac{1}{l}-\frac{\alpha^{2}}{8 n} l-\frac{1}{24 n \sqrt{n}} \frac{2 \pi l^{2}}{l^{3}}\right] .
$$

In a similar way, expanding in powers of $\frac{1}{d}$, we find,

$$
<\psi_{C}\left|\Delta H_{2}\right| \psi_{C}>\cong \frac{C_{2} e^{2}}{4 \pi \epsilon_{0}}\left[\frac{1}{d}-\frac{\alpha^{2} l^{4}}{2 d^{3}}-\frac{2 \pi l^{2}}{3} \frac{1}{d^{3}}\right] .
$$

Collecting all the term, we finally obtain,

$$
\begin{gathered}
\Delta E_{C}=<\psi_{C}\left|\Delta H_{1}+\Delta H_{2}\right| \psi_{C}> \\
\cong N \frac{e^{2}}{4 \pi \epsilon_{0}}\left[C_{1}\left\{\frac{1}{2 \sqrt{n}} \frac{1}{l}-\frac{\alpha^{2}}{8 n} l-\frac{1}{24 n \sqrt{n}} \frac{1}{l^{3}} 2 \pi l^{2}\right\}+C_{2}\left\{\frac{1}{d}-\frac{\alpha^{2} l^{4}}{2 d^{3}}-\frac{2 \pi l^{2}}{3 d^{3}}\right\}\right],
\end{gathered}
$$

where $N$ indicates the total number of electrons in the system. The system energy is proportional to $N$ since we have exploited the (non-interacting) quasi-particle picture.

Before proceeding further, a comment regarding our large $n$ assumption in (23) is pertinent. It may appear odd that our result, instead of being valid for small $n$ near ground state, is better suited for large $n$. However, one has to remember that it is not simply the Coulomb energy in a two particle system that we are after. Quite obviously the Landau level wavefunctions that we have exploited are not the natural ones for such an analysis. On the contrary, our aim is to simulate the behavior of charges in a $\mathrm{QH}$ (many body) system, subjected to Coulomb interaction. In fact, our large $n$ restriction seems to be in order if we keep in mind the fact that large $n$ means the oscillator states are more localized and there is less of overlap. This is in agreement with our model of the weakly coupled bilayer QH system having product wavefunctions in the compound state.

For $\alpha=0$ and large $n$, the leading terms in $\Delta E_{C}$ are,

$$
\Delta E_{C} \cong \frac{e^{2} N}{4 \pi \epsilon_{0}}\left[C_{1}\left(\frac{1}{2 \sqrt{n}} \frac{1}{l}\right)+C_{2}\left(\frac{1}{d}-\frac{2 \pi l^{2}}{3 d^{3}}\right)\right] .
$$

Let us try to compare our findings with the recent field theoretic computations of the Coulomb energy in bilayer quantum Hall state, as obtained by Ezawa et.al. [7]. The Coulomb energy in ground state, for large $\frac{d}{\sqrt{2} l}$, is (see equations (3.4)-(3.8) of [7] ),

$$
E_{C} \cong \frac{e^{2}}{4 \pi \epsilon_{0}} \frac{N}{4}\left[\frac{1}{l}+\frac{1}{d}-\frac{l^{2}}{d^{3}}\right] .
$$

Comparing with (29) we find that in our result (28), the relevant parameters, i.e. $N, l$ and $d$ have appeared correctly in our expression with proper signs. This concludes our analysis of the IQHE in a quantum mechanical toy model describing the bilayer system.

\section{Bilayer QH system in noncommutative space:}

Let us now analyze what happens if the noncommutativity in the space coordinates is switched on. In particular, we wish to study how it affects the Coulomb interaction. The $\mathrm{NC}(\tilde{x}, \tilde{y})$ plane obey the following algebra:

$$
[\tilde{x}, \tilde{y}]=i \theta .
$$

A representation of this structure in terms of commuting $(x, y)$ coordinates is,

$$
\tilde{x} \equiv x-\frac{\theta}{2} p_{y}, \quad \tilde{y} \equiv y+\frac{\theta}{2} p_{x} .
$$


This means that one can replace the monolayer Hamiltonian $H$ by its $\mathrm{NC}$ analogue $\tilde{H}$,

$$
H_{n c}=\frac{1}{2 m}\left[\left((1-\kappa) p_{x}-\frac{e B}{2} y\right)^{2}+\left((1-\kappa) p_{y}-\frac{e B}{2} x\right)^{2}\right]+e E\left(x-\frac{\theta}{2} p_{y}\right)
$$

where $\kappa=\frac{e \theta B}{4}$. Diagonalization of $\tilde{H}$ yields (see [9] for details) the energy spectrum,

$$
\tilde{E}=\frac{\tilde{\omega}}{2}(2 n+1)-\gamma \lambda_{+} \alpha-\frac{m}{2} \lambda_{-}^{2}
$$

with

$$
\lambda_{ \pm}=\frac{m c E}{B} \pm \frac{e m E \theta}{4 \gamma}
$$

with $\tilde{\omega}=\gamma \omega$ and $\gamma=1-\kappa$. For reasons that will become apparent later, we will consider a generalization of the above form of noncommutativity:

$$
\left[\tilde{x}_{1}, \tilde{y}_{1}\right]=i \theta_{1}, \quad\left[\tilde{x}_{2}, \tilde{y}_{2}\right]=i \theta_{2} .
$$

Thus we are taking $\theta$ to be different for the two planes. This is in effect a restricted form of non-constant or space dependent $\theta$. This form of $\theta$ has appeared in the literature. In our model, the energy for the compound state will be,

$$
\tilde{E}_{C}=\frac{\omega}{2}\left[\left(2 n_{1}+1\right) \gamma_{1}+\left(2 n_{2}+1\right) \gamma_{2}\right]-\alpha\left[\gamma_{1} \lambda_{1+}+\gamma_{2} \lambda_{2+}\right]-\frac{m}{2}\left[\lambda_{1-}^{2}+\lambda_{2-}^{2}\right] .
$$

The notation in the above is self-explanatory.

It is straightforward to consider the NC effects on the energy due to the Coulomb interaction term. Instead of that, let us study the effect of the NC-Coulomb term on conductivity. Remember that the Coulomb interaction had no effect on the conductivity in the commutative plane. In the present case, $\Delta H_{1}$ will remain unchanged since it refers to the same plane and depends on the relative position. However, $\Delta H_{2}$ will be modified since it pertains to two different planes and will depend on

$$
\begin{aligned}
{\left[d^{2}+\left(\tilde{x}_{1}-\tilde{x}_{2}\right)^{2}+\left(\tilde{y}_{1}-\tilde{y}_{2}\right)^{2}\right] } & =\left[d^{2}+\left\{x_{1}-x_{2}-\frac{1}{2}\left(\theta_{1} p_{1 y}-\theta_{2} p_{2 y}\right)\right\}^{2}\right. \\
& \left.+\left\{y_{1}-y_{2}+\frac{1}{2}\left(\theta_{1} p_{1_{x}}-\theta_{2} p_{2_{x}}\right)\right\}^{2}\right] .
\end{aligned}
$$

Thus NC effect in the conductivity operator in the compound state will be given by,

$$
\begin{aligned}
{\left[\Delta H_{n c},\left(x_{1}+x_{2}\right)\right] } & =\frac{i C_{2} e^{2}}{8 \pi \epsilon}\left(\theta_{1}-\theta_{2}\right)\left[d^{2}+\left(\tilde{x}_{1}-\tilde{x}_{2}\right)^{2}+\left(\tilde{y}_{1}-\tilde{y}_{2}\right)^{2}\right]^{-3 / 2} \\
& \times\left(\theta_{1} p_{1 x}-\theta_{2} p_{2 x}\right)
\end{aligned}
$$

and

$$
\begin{aligned}
{\left[\Delta H_{n c},\left(y_{1}+y_{2}\right)\right] } & =\frac{i C_{2} e^{2}}{8 \pi \epsilon}\left(\theta_{1}-\theta_{2}\right)\left[d^{2}+\left(\tilde{x}_{1}-\tilde{x}_{2}\right)^{2}+\left(\tilde{y}_{1}-\tilde{y}_{2}\right)^{2}\right]^{-3 / 2} \\
& \times\left(\theta_{1} p_{1 y}-\theta_{2} p_{2 y}\right)
\end{aligned}
$$

This operator correction is of higher order in $\theta$. But what is more interesting is that in this case the QH effect is lost since both the Hall currents are present. The expectation value of these correction terms are not reproduced here. Since this effect depends on $\theta_{1}-\theta_{2}$ it will vanish for $\theta_{1}=\theta_{2}$.

If $\theta_{1}=\theta_{2}$ then from the above equations we can say that there is no change in the conductivity. But if the two planes have different parameters of noncommutativity then there is some change. Possibility of having different values of $\theta$ in a single system has been considered in [12].

To conclude, in the present work, we have constructed a quantum mechanical toy model that can capture the effects of Coulomb interaction in a quantum Hall system. Our model simulates a bilayer 
quantum Hall system in the compound state and our results, (such as energy spectrum), agrees qualitatively with those of Ezawa et.al. 7]. The Coulomb term does not affect the Hall conductivity. Following the ideas of Dayi and Jellal [9], that fractional QH effect might be interpreted as integer QH effect in a noncommutative plane, we extend our bilayer model in noncommutative coordinates. We show that the noncommutative extension of the Coulomb term differs from its commutative counterpart in a higher order in $\theta$ and that too only if $\theta$ varies from layer to layer. As a future work, our aim is to extend our model to include spin effects in to account.

Acknowledgement: We thank the Referee for the constructive comments.

[1] Quantum Hall Effects Field Theoretical Approach and Related Topics, Z.F.Ezawa (World Scientific) (2000); M.Stone, (Editor), Quantum Hall Effect, World Scientific, 1992.

[2] R.B.Laughlin, Phys. Rev. Lett. 501395 (1983)

[3] F.D.M. Haldane, Phys. Rev. Lett. 51605 (1983).

[4] B.Basu and P.Bandyopadhyay, Int. J. Mod. Phys. B 12, 2649 (1998).

[5] B.Basu and P.Bandyopadhyay, Phys. Lett. A 337, 81 (2005)

[6] W.Pan, H.L.Störmer, D.C.Tsui, K.W.Baldwin and K.W.West, Phys. Rev. Lett. 90, 016801 (2003).

[7] Z. F.Ezawa, M.Eliashvili and G.Tsitsishvili, cond-mat/0501476 (to appear in Phys.Rev.B).

[8] N.Seiberg and E.Witten, JHEP 9909(1999)032. For reviews see for example M.R.Douglas and N.A.Nekrasov, Rev.Mod.Phys. 73977 (2001).

[9] O.F.Dayi and A.Jellal, J. Math. Phys. 434592 (2002).

[10] S.Ghosh, Nucl.Phys. B670 359 (2003); Phys.Lett. B 563112 (2003). For a review and references, see S.Ghosh, Noncommutative Planar Field Theories, in Progress in Field Theory Research, F.Columbus (Editor), Nova Science Publishers, Inc., U.S.A., (2004).

[11] See section 9 of the book by Ezawa in [1].

[12] A.Jellal, Electrons-Holes on Noncommutative Plane and Hall Effect, hep-th/0207269 